Preface

\title{
Recent Advances in Thrombosis and Hemostasis - Part I
}

\author{
Sam Schulman, MD, $\mathrm{PhD}^{1,2}$ Md. Shahidul Islam, MD, $\mathrm{PhD}^{3,4}$ \\ ${ }^{1}$ Thrombosis and Atherosclerosis Research Institute, and Department \\ of Medicine, McMaster University, Hamilton, \\ ON, Canada \\ 2 Department of Medicine, Karolinska Institutet, Stockholm, Sweden \\ ${ }^{3}$ Department of Clinical Science and Education, Södersjukhuset, \\ Karolinska Institutet, Stockholm, Sweden \\ ${ }^{4}$ Uppsala University Hospital, Department of Internal Medicine and \\ Emergency Medicine, Uppsala, Sweden
}

Semin Thromb Hemost 2016;42:805-807.

The burden of thrombotic diseases is heavy on the world population. It is widely known that coronary artery disease is a major cause of death but many laymen do not know that the mortality is mediated by thrombosis on a ruptured plaque. It is also less well-known that stroke is commonly the result of a thrombus that might have embolized from the heart or the carotid artery or was sometimes generated in situ. Together, ischemic heart disease and stroke account for $25 \%$ of the deaths worldwide. ${ }^{1}$ Still, deaths related to venous thromboembolism are often hidden in the statistics. With the drastically decreasing rate of autopsies performed, an increasing number of cases with fatal pulmonary embolism are misclassified. For the survivors of venous thromboembolism there is a high risk of long-term sequelae, mainly as postthrombotic syndrome and, for a smaller proportion of those with pulmonary embolism, the development of chronic pulmonary hypertension is a feared complication. Furthermore, approximately $50 \%$ of those with unprovoked venous thromboembolism will have one or several recurrent events during their remaining life with or without hospitalizations and with accumulating risk of sequelae. Venous thromboembolism is therefore associated with significant morbidity and mortality.

Despite substantial advances in the diagnosis, treatment and prophylaxis of venous thromboembolism during the past decades there is still room for improvement. It is our pleasure to provide the readers of Seminars in Thrombosis $\mathcal{E}$ Haemostasis with an update on several of these aspects in the current and a forthcoming issue of the journal. The topics were formulated by a group of leading scientists in the field, as facilitated by a gathering in Stockholm, Sweden, in November 2016, as hosted by the Swedish Society of Medicine in cooperation with Karolinska Institutet.

There seems to be an ever-expanding list of risk factors for venous thromboembolism, and they can be classified as environmental or genetic. The genome-wide association studies have verified much of what we already knew about

Address for correspondence Sam Schulman, MD, PhD, Thrombosis Service, HHS-General Hospital, 237 Barton Street East, Hamilton, ON, L8L 2X2, Canada (e-mail: schulms@mcmaster.ca). components in the coagulation cascade and the ABO blood groups and their influence on risk. Interestingly, also some single nucleotide polymorphisms in genes without apparent relation to these systems have been identified as susceptibility loci. This may teach us more about mechanisms involved in thrombus formation. Crous-Bou et al review all these risk factors and some of their interactions in the first paper of this issue. ${ }^{2}$ Familial aggregation or family history of venous thromboembolism encompasses both genetic and environmental components and thus demonstrates the characteristics of a complex trait. Zöller et al have carefully analyzed whether familial aggregation can be considered a causal risk factor for venous thromboembolism by examining each of the nine Hill's criteria for causation. $^{3}$ There are good reasons why family history always should be evaluated in the work-up of patients with venous thromboembolism.

The diagnosis of deep vein thrombosis is now almost exclusively performed with ultrasonography. Similarly, computed tomography of pulmonary arteries (CTPA) has become standard for diagnosis of pulmonary embolism because of the around the clock availability at most emergency departments. It is also perceived as a simplification, being a single exam, compared with the combined ventilation-perfusion lung scan. The latter has, however, improved its performance with the use of three-dimensional single photon emission computed tomography, abbreviated as V/Q SPECT. This method is now outperforming the CTPA in some aspects. It can also be combined with a low radiation CTPA. Hess et al present here a systematic review and meta-analysis of the performance of current diagnostic imaging methods for pulmonary embolism. ${ }^{4}$ Subsequently, they develop a thorough discussion with a review of each method as well as their role in specific patient populations.

Patients with deep vein thrombosis and to a larger extent those with pulmonary embolism are still treated in the
Copyright $\odot 2016$ by Thieme Medical Publishers, Inc., 333 Seventh Avenue, New York, NY 10001, USA.

Tel: +1(212) 584-4662.
DOI http://dx.doi.org/ 10.1055/s-0036-1593544. ISSN 0094-6176.
Thrombosis and Hemostasis -

Part I; Guest Editors: Sam

Schulman, MD, PhD, and Md.

Shahidul Islam, MD, PhD. 
hospital for several days. This is more common in the United States due to medicolegal considerations but comes at an increased cost. White et al review here the literature on outpatient treatment of pulmonary embolism. ${ }^{5}$ They then proceed to describe how an outpatient treatment protocol was developed and established at Massachusetts General Hospital in Boston. They used several strategies to overcome the psychological barriers against sending low-risk patients directly home. These included a refinement of a commonly used risk stratification score by addition of some clinically important parameters. Furthermore, they arranged for a very early follow-up mechanism at their institution. It will be interesting to see if the authors can disseminate this protocol to other United States hospitals and achieve a substantial reduction of the proportion of patients hospitalized for pulmonary embolism.

At the same hospital another initiative was taken for the patients with pulmonary embolism considered to be at intermediate or high risk for adverse outcomes. The Pulmonary Embolism Response Team (PERT) is described in a paper by Witkin et al. ${ }^{6}$ They also review the different treatment modalities that are available for the routine cases as well as the very complex or high-risk patients. Adjunctive therapies are also described. The authors conclude by reporting data on the performance of PERT and how many other centers in the United States have established similar teams.

Many patients with pulmonary embolism have severe pleuritic pain that is quite resistant to the treatment provided. The cause is typically a pulmonary infarction in one or several peripheral areas of the lung, whereby the pleura is engaged. Miniati informs the readers here on the pathology, clinical presentation, natural course, and predictors of pulmonary infarction. ${ }^{7}$ The review is beautifully illustrated with chest radiographs and CTPA films that help us realize some of the misconceptions that exist regarding diagnosis of this clinical entity.

Patients undergoing craniotomy for resection of brain tumors are at risk for postoperative thrombotic complications. Due to fear of postoperative bleeding these patients often receive suboptimal prophylaxis against venous thromboembolism after craniotomy. It is easy to understand the high thrombotic risk situation, considering the combination of cancer-associated hypercoagulability, similar effects due to treatment with steroids and/or chemotherapy, long operative procedures with complete immobilization and also, in some patients, neurological deficits that delay mobilization and recovery after surgery. Cote et al present here, using the United States National Surgical Quality Improvement Program, an analysis of venous thromboembolism after craniotomy for brain tumors. $^{8}$ They confirm that the risk is substantial and provide a thorough analysis of the risk factors for venous thromboembolism in this population.

Among the patients with brain tumors, those with highgrade glioma have probably the most pronounced risk of venous thromboembolism and inadequate prophylaxis after surgery can be detrimental. In the second paper by Cote et al, the risk of venous thromboembolism in patients with high-grade glioma and how this complication is associated with worse prognosis is reviewed. ${ }^{9}$ Prophylaxis after surgery needs to be effective, utilizing both mechanical devices and, as soon as it is safe, a pharmacological agent because thrombosis (not bleeding) is the major concern for these patients.

Occult malignancy is always in our minds when diagnosing a patient with unprovoked venous thromboembo$\mathrm{lism}$. It therefore seems reasonable to perform an extensive work-up to try and detect the cancer early, hopefully before it has metastasized and while it can still be radically removed. There is now accumulating evidence that such investigations, which are costly and not entirely harmless, will not have any significant influence on prognosis. A recent randomized trial comparing comprehensive computed tomography of the abdomen and pelvis, biphasic enhancement of the liver, parenchymal pancreatography as well as uniphasic enhancement of distended bladder versus limited, age- and sex-appropriate screening did not demonstrate any significant differences in outcomes and prognosis. ${ }^{10}$ Prandoni et al present here for the first time results of a similar randomized trial, in which they compared computed tomography of thorax, abdomen, and pelvis plus test for fecal occult blood versus limited screening based on the judgement of the physician in combination with preferences of the patient. ${ }^{11}$ The study was stopped for feasibility reasons but the results support the concept that "less is more."

In the concluding review by Schulman, the main novelties in the management of venous thromboembolism are discussed with reference to recent meta-analyses, systematic reviews, and updates in international guidelines. ${ }^{12}$ The topics reviewed are risk stratification for patients with pulmonary embolism, thrombolytic therapy for pulmonary embolism or deep vein thrombosis, options for initial anticoagulant treatment (where the non-vitamin $\mathrm{K}$ antagonist oral anticoagulants now have become the suggested first choice but they are not suitable for all patient populations), duration of anticoagulation, risk stratification for recurrent venous thromboembolism, the questionable need to treat minimal thromboembolism, and finally whether to use compression stockings to prevent postthrombotic syndrome.

It is our hope that readers of Seminars in Thrombosis $\mathcal{E}$ Haemostasis will enjoy these review articles, some of which also contain original data. The papers should support improvements in clinical management of venous thromboembolism and also stimulate to new studies for better understanding of the disease mechanism or to the advancement in quality of care. In clinical practice, the physicians will still have to make many difficult decisions in individual cases when solid evidence is lacking. Some of those challenges may also result in important research projects that will eventually inform clinicians. We also look forward to providing the second issue ('Part 2') in this series in the near future. 


\section{References}

1 Raskob GE, Angchaisuksiri P, Blanco AN, et al; ISTH Steering Committee for World Thrombosis Day. Thrombosis: a major contributor to global disease burden. Semin Thromb Hemost 2014;40(7):724-735

2 Crous-Bou M, Harrington LB, Kabrhel C. Environmental and genetic risk factors associated with venous thromboembolism. Semin Thromb Hemost 2016;42(8):808-820

3 Zöller B, Li X, Ohlsson H, et al. Epidemiology of familial aggregation of venous thromboembolism. Semin Thromb Hemost 2016;42(8): 821-832

4 Hess S, Frary EC esq, Gerke O, Madsen PH. State-of-the-art imaging in pulmonary embolism: Ventilation/perfusion single-photon emission computed tomography versus computed tomography angiography - controversies, results, and recommendations from a systematic review. Semin Thromb Hemost 2016;42(8):833-845

5 White B, Rosovsky R, Parry BA, Kabrhel C. The outpatient treatment of venous thromboembolism: operational impact and the role of novel anticoagulants. Semin Thromb Hemost 2016;42(8): 846-856
6 Witkin AS, Harshbarger S, Kabrhel C. Pulmonary embolism response teams. Semin Thromb Hemost 2016;42(8):857-864

7 Miniati M. Pulmonary infarction: an often unrecognized clinical entity. Semin Thromb Hemost 2016;42(8):865-869

8 Cote DJ, Dubois HM, Karhade AV, Smith TR. Venous thromboembolism in patients undergoing craniotomy for brain tumors: a U.S nationwide analysis. Semin Thromb Hemost 2016;42(8):870-876

9 Cote DJ, Dawood HY, Smith TR. Venous thromboembolism in patients with high grade glioma. Semin Thromb Hemost 2016; 42(8):877-883

10 Carrier M, Lazo-Langner A, Shivakumar S, et al; SOME Investigators. Screening for occult cancer in unprovoked venous thromboembolism. N Engl J Med 2015;373(8):697-704

11 Prandoni P, Bernardi E, Dalla Valle F, et al. Extensive computed tomography versus limited screening for detection of occult cancer in unprovoked venous thromboembolism: a multicenter, controlled, randomized clinical trial. Semin Thromb Hemost 2016; 42(8):884-890

12 Schulman S. Update on the treatment of venous thromboembolism. Semin Thromb Hemost 2016;42(8):891-898 